\title{
The Dedicated ICRH System for the Stellarator Wendelstein 7-X
}

\author{
J.Ongena, A.Messiaen, P.Dumortier, F.Durodie, Ye.O.Kazakov, F.Louche, \\ B.Schweer, M.Vervier, D.Van Eester, \\ R.Koch, A.Krivska, A.Lyssoivan, M.Van Schoor, T.Wauters \\ Laboratory for Plasmaphysics, Ecole Royale Militaire - Koninklijke Militaire School, \\ TEC Partner, Brussels, Belgium \\ V.Borsuk, O. Neubauer, O. Schmitz \\ IEK-4 / Plasmaphysik, Forschungszentrum Juelich, TEC Partner, Juelich, Germany \\ G.Offermans \\ ZEA-1, Forschungszentrum Juelich, Juelich, Germany
}

\author{
Y. Altenburg, C.Baylard, D.Birus, S.Bozhenkov, D.A. Hartmann, J.Kallmeyer, S. \\ Renard, R.Wolf \\ Max-Planck Institute für Plasmaphysik, Teilinstitut Greifswald, Greifswald, Germany
}

\begin{abstract}
The current status of the mechanical and electromagnetic design for the ICRF antenna system for W7-X is presented. Two antenna plugins are discussed : one consisting of a pair of straps with pre-matching to cover the first frequency band 25-38 $\mathrm{MHz}$ and a second one consisting of two short strap triplets to cover a frequency band around $76 \mathrm{MHz}$. This paper focusses on the two strap antenna for the lower frequency band. Power coupling of the antenna to a reference plasma profile is studied with the help of the codes TOPICA and Microwave Studio, that deliver the scattering matrix needed for the optimization of the geometric parameters of the straps and antenna box. Radiation power spectra for different phasings of the two straps are obtained using the code ANTITER II and different heating scenarii are discussed. The potential for heating, fast particle generation and current drive is discussed. The problem of RF coupling through the plasma edge and of edge power deposition is summarized. The system contains a prematching capacitor to limit the maximum voltage in the system, and the large mutual coupling between the 2 straps is counterbalanced by the use of a decoupler. The mechanical design highlights the challenges encountered with this antenna : adaptation to a large variety of plasma configurations, the limited space within the port to accommodate the necessary matching components and the watercooling needed for long pulse operation.
\end{abstract}

Keywords: ICRH, W7-X. coupling, matching, decoupler, 5-ports junction

PACS: $28.52 \mathrm{Cx}, 52.50 \mathrm{Qt}, 52.55 \mathrm{Hc}$

\section{INTRODUCTION}

The Wendelstein 7-X (W7-X) stellarator (average minor and major radius a $=0.55 \mathrm{~m}$ and $\mathrm{R}_{0}=5.5 \mathrm{~m}$, average magnetic field on axis 2.5T) with superconducting coils, under construction in Greifswald, will be the first "fully-optimized" stellarator [1]. It will be the largest device of this class in the world. The mission of the project is to demonstrate the reactor potential of the optimized stellarator line [2]. To demonstrate that reactor relevant plasma parameters can be achieved in steady-state, the W7-X experiment is designed for plasma pulses with 30 minutes duration at a heating power of $10 \mathrm{MW}$, with Electron Cyclotron Resonance Heating (ECRH) at $140 \mathrm{GHz}$ as main heating system. This is complemented by Neutral Beam Injection (NBI) and Ion Cyclotron Resonance Heating (ICRH) for shorter pulse durations (up to 10s).

This paper presents main features of the ICRH system that is currently under design for W7-X. Aiming at a power of $\sim 1$ MW such a system provides a heating and current drive tool complementing the steady-state ECRH for short periods. It will provide central plasma heating and fast ion production at very high plasma densities. It also has potential for electron current drive with the aim to assist in studying fast particle confinement at high beta and Alfvén instabilities and can provide Ion Cyclotron Wall Conditioning (ICWC). The advantage of ICRH is the absence of a cut-off at high density, allowing applications beyond the capabilities of standard ECRH in the magnetic topology of a stellarator. 
The ICRH antenna is intended to operate at the main magnetic fields $\left(\mathrm{B}_{\mathrm{o}}=2.5 \mathrm{~T}\right)$ foreseen for operation of the $140 \mathrm{GHz}$ ECRH system. Operation at $1.25 \mathrm{~T}$ is another possibility, but then mainly without ECRH. Initially a feasability study was conducted to design an antenna for operating over the whole frequency range $25-76 \mathrm{MHz}$ to cover a large range of ICRF scenarios. It was found that with the capacitors available for installation in the limited space of the port such prematching cannot be achieved over the whole frequency range. Therefore two antenna plug-ins are proposed: a first antenna plug-in to cover the frequency band $25-38 \mathrm{MHz}$ for $\mathrm{H}$ or ${ }^{3} \mathrm{He}$ minority heating at $\omega=\omega_{\mathrm{ci}}$ and a second one for operation in a frequency band with $76 \mathrm{MHz}$ as main frequency for $\omega=2 \omega_{\mathrm{cH}}$ minority heating. These antennas are proposed to be interchangeable, i.e. the outside connections will remain the same, and only the parts internal to the port will need to be exchanged, as will be discussed below. The matching components, however, will have to be adapted to the new frequency band, but are outside the torus hall. For the first frequency band the TEXTOR generators and RF hardware can be used. The second one can be implemented depending upon the availability of the necessary generator at $76 \mathrm{MHz}$. The selected antenna configurations to meet the foreseen applications and the narrow space available in the port at W7-X is: (i) a pair of long straps for the $25-38 \mathrm{MHz}$ band and (ii) a pair of triplets of short straps for the $76 \mathrm{MHz}$ band (see Fig $2 \mathrm{a}$ and $2 b$ ).

\section{ICRH SCENARIOS FOR W7-X AND POTENTIAL FOR HEATING, CURRENT DRIVE AND FAST PARTICLE GENERATION}

The main RF scenarios that can be applied at $2.5 \mathrm{~T}$ are: $\mathrm{H}$ minority heating in ${ }^{4} \mathrm{He}$ or $\mathrm{D}(38 \mathrm{MHz})$, and ${ }^{3} \mathrm{He}$ minority heating in $\mathrm{H}(25 \mathrm{MHz})$ and $2^{\text {nd }}$ harmonic $\mathrm{H}$ heating in ${ }^{4} \mathrm{He}$ or $\mathrm{D}(76 \mathrm{MHz})$. Other possible scenarios are $2^{\text {nd }}$ harmonic $\mathrm{D}$ in $\mathrm{H}(38 \mathrm{MHz}),{ }^{3} \mathrm{He}$ minority heating in ${ }^{4} \mathrm{He}$ or $\mathrm{D}(25 \mathrm{MHz})$. At a toroidal field of $1.25 \mathrm{~T}$ the following scenarios are applicable: $2^{\text {nd }}$ harmonic ${ }^{3} \mathrm{He}$ in $\mathrm{H}$, D or ${ }^{4} \mathrm{He}(25 \mathrm{MHz}), 2^{\text {nd }}$ harmonic $\mathrm{H}$ heating in ${ }^{4} \mathrm{He}$ or $\mathrm{D}(38 \mathrm{MHz})$ and possibly also $3^{\text {rd }}$ harmonic ${ }^{3} \mathrm{He}$ heating in ${ }^{4} \mathrm{He}$ or $\mathrm{D}(38 \mathrm{MHz})$.

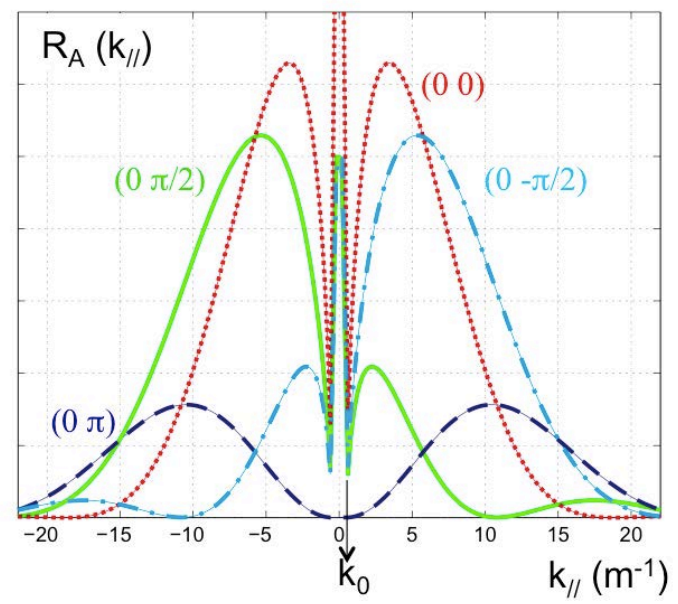

FIGURE 1: Antenna $\mathrm{k}_{/ /}$power spectrum as calculated by ANTITER II. Dotted ( $\left.\begin{array}{ll}0 & 0\end{array}\right)$ phasing for ICWC applications, full and dot-dashed curves to $(0 \pm \pi / 2)$ phasing for current drive applications, and the dashed curve to $(0 \pi)$ phasing for plasma heating.
The problem of coupling through the plasma edge and radiated power spectrum has been analysed, to a first approximation [3], by means of the analytical coupling code ANTITER II [4] for the different relative phasings of the two straps considered for the antenna system discussed in this paper: $(00),(0 \pm \pi / 2),(0 \pi)$. This code uses a Fourier analysis in $\left(\mathrm{k}_{\mathrm{z}}, \mathrm{k}_{\mathrm{y}}\right)$ space (z-axis along the toroidal field $\mathrm{B}, \mathrm{y}$-axis perpendicular to the $\mathrm{z}$-axis and radial axis) assuming a simplified antenna geometry (recessed antenna box in the device wall with 2 thin straps [3]), which is close to the intended final antenna geometry. The antenna $\mathrm{k}_{/ /}$power spectrum launched at the antenna obtained with ANTITER II is given in Fig. 1 using the reference density profile provided by the W7-X team. The $\left|\mathrm{k}_{/ /}\right|$values at the peak of the radiated power correspond to $\sim 11$ $\mathrm{m}^{-1}$ for $(0 \pi), \sim \pm 6.5 \mathrm{~m}^{-1}$ for $(0 \pm \pi / 2)$ and $\sim 2.5 \mathrm{~m}^{-1}$ for $(00)$ phasing.

Highest coupling occurs for monopole phasing $\left(\begin{array}{ll}0 & 0\end{array}\right)$ but this phasing also leads to significant excitation of coaxial and surface modes for parallel wave numbers $\left|\mathrm{k}_{/ /}\right|<\mathrm{k}_{0}$ ( $\mathrm{k}_{0}$ being the wave number in vacuum) with power absorption into the plasma edge. Dipole $(0 \pi)$ phasing can be used for heating, but best coupling is obtained for the $(0 \pm \pi / 2)$ phasing, due to the much higher excitation of a lower $|\mathrm{k} /|$ at about $6.5 \mathrm{~m}^{-1}$ (see Fig. 1); the wave tunneling region before reaching the wave cut-off inside the plasma edge is shorter for $(0 \pm \pi / 2)$ phasing and this underlines again the advantage to use this phasing also for heating. As tunneling of the waves through the evanescence zone is rather sensitive to the electron density profile in front of the antenna, the results presented here will therefore have to be cross-checked with experiment. 


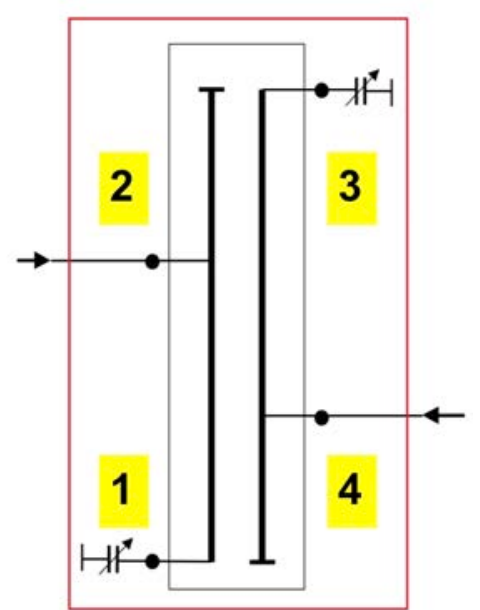

FIGURE 2a. The Antenna plug-in for 25-38 $\mathrm{MHz}$ as a 4-port junction.

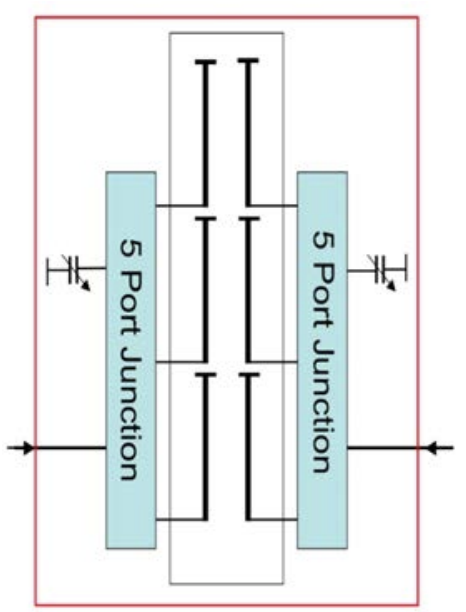

FIGURE 2b. The Antenna plug-in for the frequency band around $76 \mathrm{MHz}$.
There is a large effect if Alfvén or ion-ion hybrid resonances are located in the edge. An Alfvén resonance will be present in the edge if $\left|\mathrm{k}_{/ /}\right|>\mathrm{k}_{0}$ when $v<v_{\mathrm{c} \text {,majority }}$ (i.e. for $v<34.6 \mathrm{MHz}$ in a $\mathrm{H}\left(\mathrm{D}\right.$ or $\left.{ }^{3} \mathrm{He}\right)$ plasma and $v<17.3 \mathrm{MHz}$ in a $\mathrm{D}(\mathrm{H})$ plasma for $\mathrm{B}_{\text {edge }} \approx 2.27 \mathrm{~T}$ ). A nonnegligible fraction of the radiated power can be absorbed in the plasma edge under such conditions.

First estimates for the single transit absorption of these schemes have been obtained with the 1D code TOMCAT-U [5], taking only one toroidal mode launched in the plasma, corresponding to the

dominant peak in the $\mathrm{k}_{/ /}$spectrum. For $\mathrm{H}$ concentrations in the range of $5-15 \%$, minority heating in $\mathrm{D}$ is a very efficient heating scheme for expected high density W7-X parameters $\left(\mathrm{n}_{\mathrm{e}}=210^{20} \mathrm{~m}^{-3}, \mathrm{~T}_{0}=3 \mathrm{keV}\right)$. Inverted scenarios such as ${ }^{3} \mathrm{He}$ minority in $\mathrm{H}$ can also be used but could pose difficulties because of edge power deposition as discussed above. Estimates show that for the case of ${ }^{3} \mathrm{He}$ minority in $\mathrm{H}$ at $25 \mathrm{MHz}$ and B $=2.46 \mathrm{~T}$, between 15 and $20 \%$ of the launched power could be lost in the plasma edge resulting in a reduced efficiency of the heating scheme and possibly localized deposition of power at first wall components.

High energy tails can only be created when working at low $\left(1-210^{19} \mathrm{~m}^{-3}\right)$ densities. MeV minority $\mathrm{H}$ tails can easily be created at low density and low minority concentration provided the RF power density level is sufficiently high (calculations show that $1 \mathrm{MeV}$ ions are 10 orders of magnitude more abundant when $\mathrm{P}_{\mathrm{RF}}=1 \mathrm{MWm}^{-3}$ than at $0.2 \mathrm{MWm}^{-}$ $\left.{ }^{3}\right)$. High density operation renders tail formation hard and would require unrealistic power density levels. Current drive scenarios are realistic at low densities $\left(\sim 210^{19} \mathrm{~m}^{-3}\right)$ with efficiencies for ions of $\sim 3-4 \mathrm{kA} / \mathrm{MW}$ and for the electrons $\sim 15$ $\mathrm{kA} / \mathrm{MW}$.

Note that (a) part of the codes adopted assume equivalent tokamak magnetic geometries. The conclusions in this section are therefore of a preliminary nature and checks with other codes existing or still to be developed taking into account the real magnetic topology of the plasma will be undertaken in the future

\section{ELECTROMAGNETIC DESIGN}

The geometry of these compact antennae in the narrow port has been optimized for the best coupling conditions to a reference plasma density profile [3]. The antenna plug-in designed for operation between 25 and $\mathrm{MHz}$ consists of a pair of watercooled single straps, each of them tuned commercially available vacuum capacitor at the rear of the antenna housing box (Fig. 2a). To reduce the maximum voltage on the system, a matching has been implemented by connecting the RF transmission lines intermediate position on each poloidal strap (referred to as 'tap' in what follows), which on one side is connected to the capacitor and grounded antenna box at the other end. The second version, optimized for operation in a frequency band around the central frequency of $76 \mathrm{MHz}$, consists of a pair of poloidal strap triplets fed by a tunable 5-port junction (Fig. 2b). The 5-port junction with its tuning vacuum capacitor also placed at the rear of the antenna box in the antenna port, similar to port

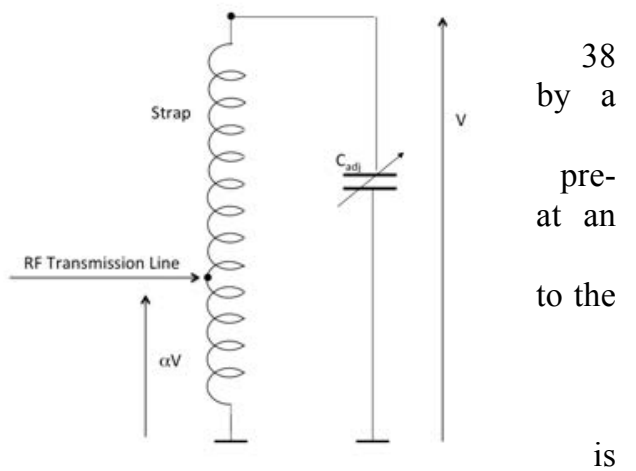

FIGURE 3. Approximate Equivalent Electrical Circuit of Strap the 4- 
junction proposed for the ICRF antenna for ITER [7]. A feeding-matching system using the two $2 \mathrm{MW}$ RF generators

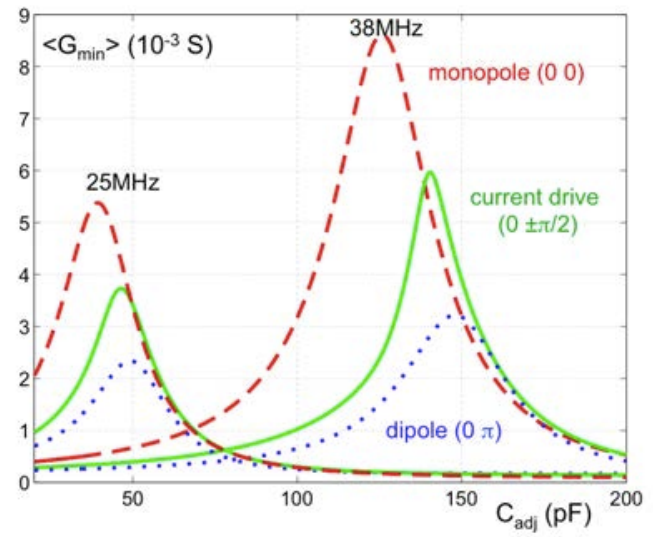

FIGURE 4. Evolution of $\mathrm{G}_{\min }$ vs. $\mathrm{C}_{\text {adj }}$ for $25 \mathrm{MHz}$ (dashed) and $38 \mathrm{MHz}$ (full). The dashed curves correspond to $\left(\begin{array}{ll}0 & 0\end{array}\right)$ phasing, the full ones to $(0 \pm \pi / 2)$ phasing and the dotted ones to $(0 \pi)$ phasing. The peak value of $\left\langle\mathrm{G}_{\min }>\right.$ determines the value $\mathrm{C}_{\mathrm{adi}, \max }$ corresponding to a tuned system.
(25-38 MHz band) of TEXTOR allows a flexible antenna phasing with as applications: $\left(\begin{array}{ll}0 & 0\end{array}\right)$ phasing for Ion Cyclotron Wall Conditioning, $(0$ $\pi)$ phasing for heating / fast particle generation and $(0 \pm \pi / 2)$ for heating with some current drive. The $(0 \pm \pi / 2)$ phasing delivers most power into the plasma due to the higher $\left|\mathrm{k}_{/}\right|$excitation, as is visible in the spectrum (Fig. 1). Other phasings are evidently possible as well. For plasma operation without ICRH, the antenna can be almost completely withdrawn into the port, away from the plasma boundary, to reduce the heat loads from the plasma and ECRH stray radiation. This paper will focus on the design of the two strap antenna for the lower frequency band 25-38 $\mathrm{MHz}$.

An approximate equivalent electrical schema of each antenna strap together with the tuning capacitor consists of an inductance in parallel with a variable capacitor, as shown in Fig. 3. For a given input voltage to the strap, the maximum voltage in the RF transmission lines is determined by the pre-matching, done by positioning the 'tap' at a fraction $\alpha \mathrm{L}$ from of the total strap inductance $\mathrm{L}$ with $0<\alpha<1$. The voltage at the position of the tap is then $\alpha \mathrm{V}$, with $\mathrm{V}$ the maximum allowable voltage in the antenna system.

The antenna box consists of a 4-port network (see Fig. 2a) which is modeled by the TOPICA [8] or MWS [9] codes. TOPICA uses the plasma density profile as provided by the W7-X team in front of the antenna. This is not possible in MWS, and instead a stratified dielectric profile [10] is used to mimic the density profile for coupling simulations and comparison of the results obtained with TOPICA. Optimisation of the design the proceeds as follows. For each variation in the geometry of the antenna box (strap width, distance between the straps, antenna box depth etc...), the $4 \times 4$ scattering matrix describing the electromagnetic behaviour of the antenna loaded by the plasma reference profile is obtained from these codes.

From the scattering matrix $\mathrm{S}$ we derive the admittance matrix $\mathrm{Y}$, which results in a system of equations 4 equations linking the voltages and currents $\mathrm{V}_{\mathrm{i}}$ and $\mathrm{I}_{\mathrm{i}}$ at the ports $1,2,3,4$. To be able to solve this system of the 8 unknowns $\mathrm{I}_{1 \ldots 4}$ and $\mathrm{V}_{1 \ldots 4}$ we impose that the amplitudes of the currents in port 1 and port 3 are equal for a chosen phasing $\Delta \phi,\left|\mathrm{I}_{1}\right|=\left|\mathrm{I}_{3}\right|$ with $\mathrm{I}_{1}=\mathrm{I}_{3} \exp (\mathrm{i} \Delta \phi)$. Then we make use of the relation between voltage and current at the capacitors : $\mathrm{I}_{1,3}=$ $\mathrm{V}_{1,3}\left(\mathrm{i} \omega \mathrm{C}_{\mathrm{adj}}\right)$. We have now a system of equations that allows to determine $\mathrm{I}_{2}, \mathrm{I}_{4}, \mathrm{~V}_{2}$ and $\mathrm{V}_{4}$ and thus also the delivered active power $\mathrm{P}_{2}$ and $\mathrm{P}_{4}$ at ports 2 and 4 of the 4-port junction. We have now to choose the value of $\mathrm{C}_{\mathrm{adj}}=\mathrm{C}_{\mathrm{adj}, \max }$ which produces the best prematching compatible with the equality $\left|\mathrm{I}_{1}\right|=\left|\mathrm{I}_{3}\right|$ and their difference in phasing. This is obtained by means of a scan in $\mathrm{C}_{\text {adj }}$ (see Fig. 4). For a given frequency and phasing of the straps, the value $C_{a d j, \max }$ is the value of $\mathrm{C}_{\text {adj }}$ for which the mean minimum conductance of feeding lines 2 and $4,<\mathrm{G}_{\min }>$ (defined as $\left\langle\mathrm{G}_{\min }\right\rangle=\left(\mathrm{G}_{\min , 2}+\mathrm{G}_{\min , 4}\right) / 2$ with $\mathrm{G}_{\text {min, },}=2 \mathrm{P}_{\mathrm{i}} / \mathrm{V}^{2}{ }_{\text {max }, \mathrm{i}}$ where $\mathrm{V}_{\text {max }, \mathrm{i}}$ is the anti-node voltage on line i) reaches a maximum, as shown on Fig. 4, showing an example where we varied the strap width.

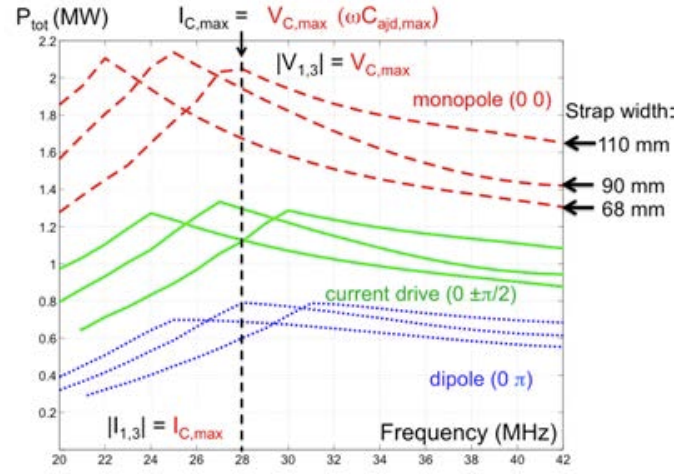

FIGURE 5: Evolution of the total coupled power $\mathrm{P}_{\text {tot }}$ versus frequency for different strap widths: $68 \mathrm{~mm}, 90 \mathrm{~mm}$ and $110 \mathrm{~mm}$. The dashed curves correspond to $\left(\begin{array}{ll}0 & 0\end{array}\right)$ phasing, the full ones to $(0 \pm \pi / 2)$ phasing and the dottted ones to $(0 \pi)$ phasing. A strap width of $90 \mathrm{~mm}$ offers best power coupling for both 25 and $38 \mathrm{MHz}$.

Repeating this procedure for the phasings of interest and all frequencies in the band then results in a set of $\mathrm{C}_{\mathrm{adj}, \mathrm{max}} \mathrm{values}$ versus frequency for the various phasings. The total active power $\mathrm{P}_{\text {tot }}=\mathrm{P}_{2}+\mathrm{P}_{4}$ can then be obtained as a function of the frequency $v$ solving the set of equations using at each frequency the corresponding value of $\mathrm{C}_{\mathrm{adj}}$,max found previously.

Due to the tap feed, the maximum voltage is occuring at the tuning capacitor. To determine the maximum power capability of the antenna system, we have to take into account the maximum voltage $V_{C \text {,max }}$ and current $I_{C \text {,max }}$ specifications of the capacitor. The limitations in power are then determined either by $V_{C, \max }$ or $I_{C, \max } /\left(\omega C_{a d j}\right)$ depending on which value is the smallest. A plot of the evolution of the maximum power versus frequency obtained in this way 


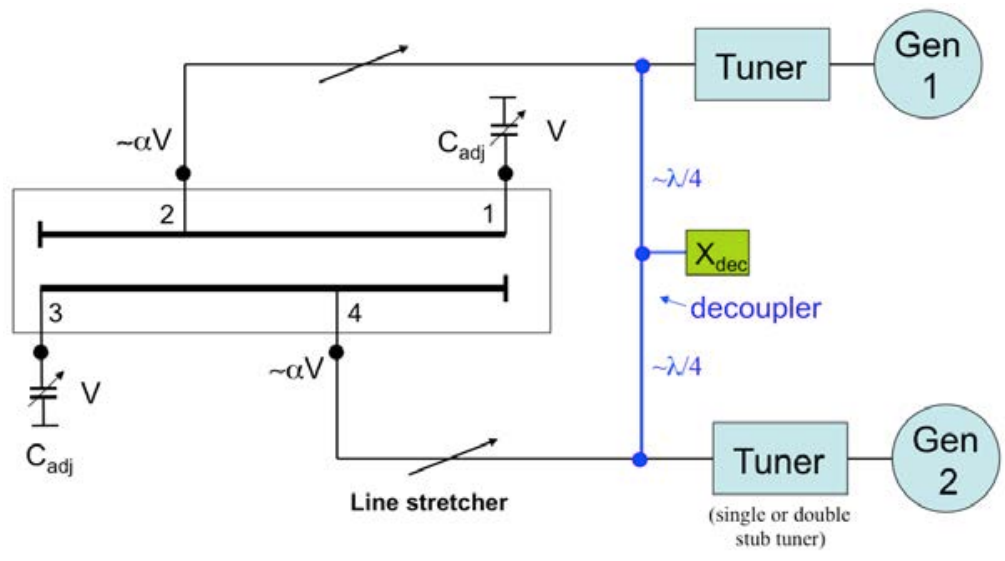

FIGURE 6: Matching and decoupler network shows a maximum, see Fig. 5 and the curve is divided in two parts : left of the maximum, limited by $\mathrm{I}_{\mathrm{C}, \max }$ (and then $\left|\mathrm{V}_{1,3}\right|$ $<\mathrm{V}_{\mathrm{C} \text {,max }}$ ) and right of the maximum, limited by $\mathrm{V}_{\mathrm{C} \text {,max }}$ (and then $\left|\mathrm{I}_{1,3}\right|<\mathrm{I}_{\mathrm{C} \text {,max }}$ ). The optimization then consists in selecting a geometry that avoids a reduction of the power at $25 \mathrm{MHz}$ and has a beneficial influence at $38 \mathrm{MHz}$. For the case of a study of strap widths (Fig. 4), that was found for a strap width of $90 \mathrm{~mm}$. The behaviour of $P_{\text {tot }}$ versus frequency $v$ at both sides of the angular point can be explained by the mean strap impedance $Z_{f}$ at the feeding points (ports 1 and 3), defined as:

$$
\mathrm{Z}_{\mathrm{f}}=1 / \mathrm{Y}_{\mathrm{f}}=\mathrm{R}_{\mathrm{f}}+\mathrm{i} \mathrm{X}_{\mathrm{f}}=2<\mathrm{P}>/\left|\mathrm{I}_{1,3}\right|^{2}
$$

with $<\mathrm{P}>=\left(\mathrm{P}_{2}+\mathrm{P}_{4}\right) / 2, \mathrm{P}_{2}$ and $\mathrm{P}_{4}$ being the power delivered to ports 2 and 4 by the feeding lines and $\mathrm{I}_{1}$ and $\mathrm{I}_{3}$ the current at ports 1 and 3. The strap conductance is defined as $\operatorname{Re}\left(\mathrm{Y}_{\mathrm{f}}\right)=\mathrm{G}_{\mathrm{f}} \sim \mathrm{R}_{\mathrm{f}} / \mathrm{X}_{\mathrm{f}}^{2}\left(\mathrm{R}_{\mathrm{f}}^{2}<<\mathrm{X}_{\mathrm{f}}^{2}\right)$. $\mathrm{P}_{\text {tot }}$ varies as $\mathrm{R}_{\mathrm{f}} \mathrm{I}_{\mathrm{C} \text {,max }}^{2} / 2$ for frequencies smaller than the angular point (which corresponds to $I_{C, \max }=V_{C, \max } \omega C_{\text {adj,max }}$ ) and as $G_{f} V_{C, \text { max }}^{2} / 2$ for higher frequencies. Note that both $\mathrm{R}_{\mathrm{f}}$ and $\mathrm{X}_{\mathrm{f}}$ increase with increasing frequency $v$ and are decreasing with the strap width but not in the same way. This results in an increase of $\mathrm{G}_{\mathrm{f}}$ with decreasing frequency and increasing strap width. Note also that with increasing strap width, the strap input reactance decreases. This then leads in addition to a higher value for the capacitance at the maximum frequency of the frequency band. In turn, this leads to capacitance values needed to tune the antenna, which are safely larger than the minimum capacitance value for commercially available capacitors, even when taking into account the decrease with frequency of the capacitor reactance due to its internal inductance. An experimental test will be undertaken during the construction of the antenna to test the effective tuning range of the capacitor and adapt if necessary the strap width.

Matching the generators to this antenna can be done with a traditional system using line stretchers and stubs (Fig. 6). Slow coupling variations can be compensated using an automatic tuning system [11]. As the straps in antenna box are closely packed there is a large mutual coupling between the straps. To minimize their effect on the matching circuit and to equalize the power supplied by both generators for $(0 \pm \pi / 2)$ phasing a decoupler system [12] is incluced.

For the upper part of the frequency band $(\sim 76 \mathrm{MHz})$ the conceptual design of another plug-in has been

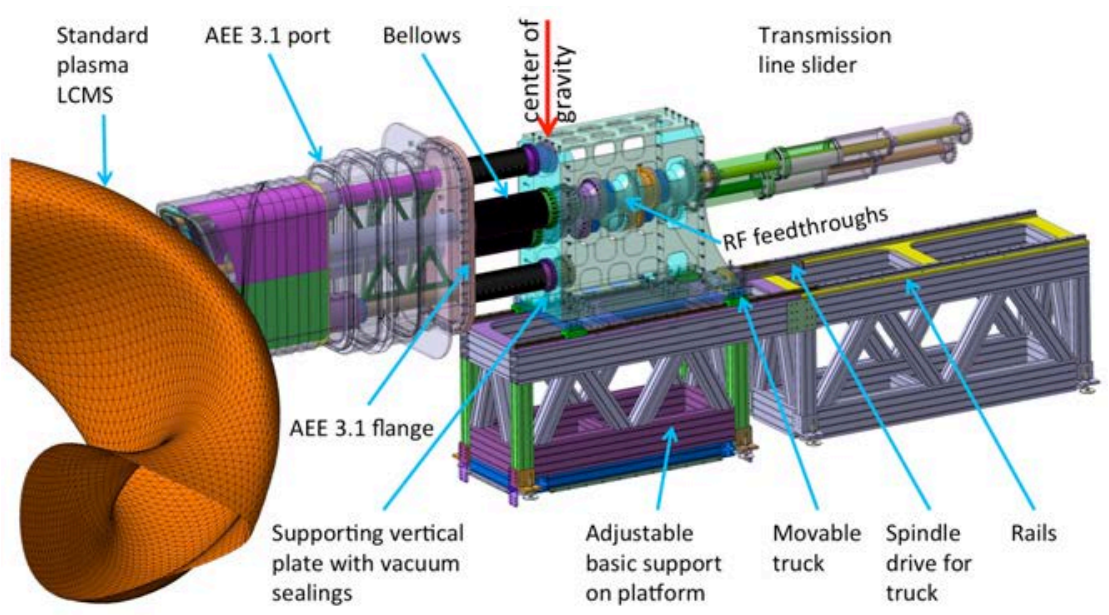

FIGURE 7 : Outline mechanical design of the antenna system for W7-X. presented. It consists of two triplets of straps, whereby the individual straps of each of the triplets is fed in parallel by means of a 5-port junction. If only one $76 \mathrm{MHz}$ generator is used, then this system will require in addition a $3 \mathrm{~dB}$ splitter/coupler together with a line stretcher to provide for a flexible phasing of the toroidal strap triplets. With two generators at $76 \mathrm{MHz}$, a matching system as described above can be used. The $76 \mathrm{MHz}$ band would allow for second harmonic $\mathrm{H}$ heating at $2.5 \mathrm{~T}$.

\section{MECHANICAL DESIGN}

The ICRH antenna designed for W7-X will be installed in the equatorial plane in module 3 at port AEE3.1. A design view on the global system with standard plasma is presented in Fig. 7. More details of the antenna system are shown in 
Fig. 8 where the shielding of the antenna box and the plasma itself are removed. The following requirements need to be integrated in the design:

- Installation of the antenna assembly in $\mathrm{W} 7-\mathrm{X}$ without in-vessel access

- Two transmission lines to deliver the ICRH power

- Optimal coupling to different plasma configurations

- Provisions to install a tuning capacitor

- Water cooling during long pulse (up to $30 \mathrm{~min}$ ) dc plasma operation

- Diagnostics for measurement of ICRH power and edge plasma conditions

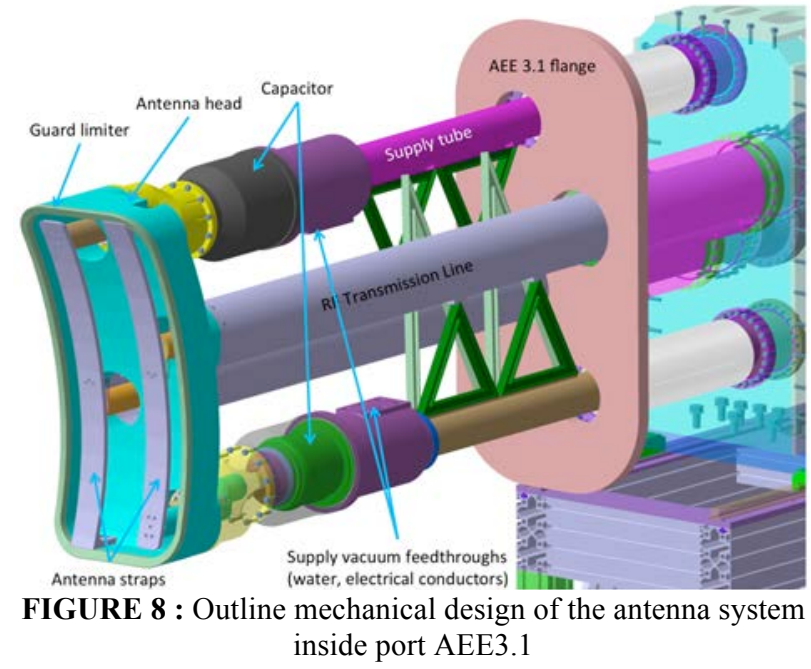

Installation of the Antenna System avoiding In-Vessel Access

The ICRH antenna is designed such that it can be integrated at a port without mechanical support from the inner vessel. Therefore the ICRH antenna unit is mounted on a table, and can be moved on pre-aligned rails. The center of gravity indicated in the principal set-up shown in Fig. 7 is located outside the AEE3.1 flange, which will allow an easy transportation from the assembly hall to the W7-X torus. The basic support structure with rails is aligned to an axis defined by the port centers of the inner (plasma) and outer (cryostat) vessel. Sufficient clearance is foreseen in the port such that the system will be able to cope with displacements of the inner vessel with respect to the cryostat port during W7-X pump down.

\section{Adapting to Different Plasma Shapes}

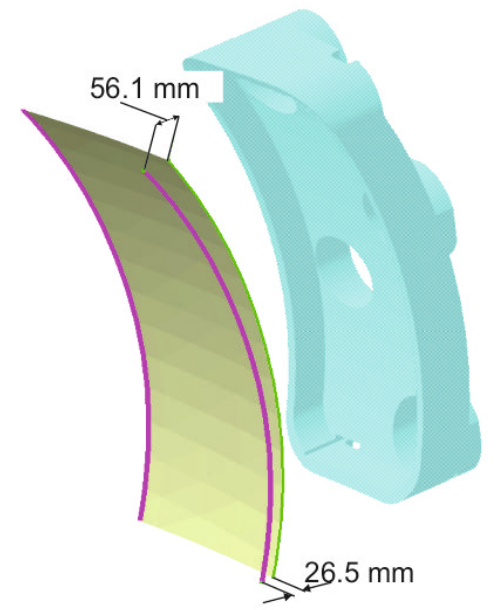

FIGURE 9 : Illustration of the poloidal and torodial curvature of antenna head and straps. The gray surface represents the front face of the antenna box. The full line at the right is rotated toroidally along the vertical axis of W7-X. The differences in the distance on the top and the bottom are shown. The numbers indicate the deviations of the poloidal shape at the rear front side of the antenna head
Different magnetic field configurations are foreseen for experiments on W7-X, each leading to a different plasma shape. The distance between the Last Closed Magnetic Surface (LCMS) and the outside edges of the antenna box in parking position for the most extreme plasma shapes can be up to $330 \mathrm{~mm}$. The shape of the antenna box and straps has been optimized for plasmas corresponding to the so-called standard field configuration. This results in an antenna box and strap surface structure with a poloidal and toroidal curvature, see Fig. 9. In this case the distance between any point on the straps and LCMS will be $50 \mathrm{~mm}$, whereby the antenna box is positioned $257 \mathrm{~mm}$ into the inner vessel with respect to the parking position of the antenna in the port. For all other configurations the width of the gap between straps and LCMS will vary over the strap surface, and this will cause a variable loading of parts of the straps and therefore the RF coupling efficiency (Fig. 10). To that end, a gas feed tube with several holes in poloidal direction along the sides of the antenna box will be implemented to maximise coupling independent of the plasma shape.

The position of the antenna box with respect to the LCMS for a given magnetic configuration of $\mathrm{W} 7-\mathrm{X}$ will also depend on the heat load from the plasma (escaping fast particles and micro wave stray radiation), which should be below $2 \mathrm{MW} / \mathrm{m}^{2}$ at the straps and edges of the antenna box, as discussed below.

The antenna is mounted on a movable truck, which allows a change of 
position with a velocity of $1 \mathrm{~mm} / \mathrm{s}$ even during plasma operation. A mechanical decoupling from the outer vessel is achieved by edge welded bellows covering the RF vacuum transmission lines.

\section{Cooling Conditions for Long Pulse Plasma Operation}

A challenging task in the antenna design is the integration of the cooling circuits. The resistive losses of the RF power in transmission lines and antenna box for durations of about $20 \mathrm{~s}$ must be removed. A total maximum power loss of $200 \mathrm{~kW}$ mostly deposited in the straps and antenna box was assumed for both circuits. Therefore the straps and the antenna box must be actively cooled by two water circuits. Inside the transmission lines in vacuum the temperature rise is tolerable even for the most critical duty cycle of $20 \mathrm{~s}$ "on" to $120 \mathrm{~s}$ "off" and they therefore need no active cooling. In
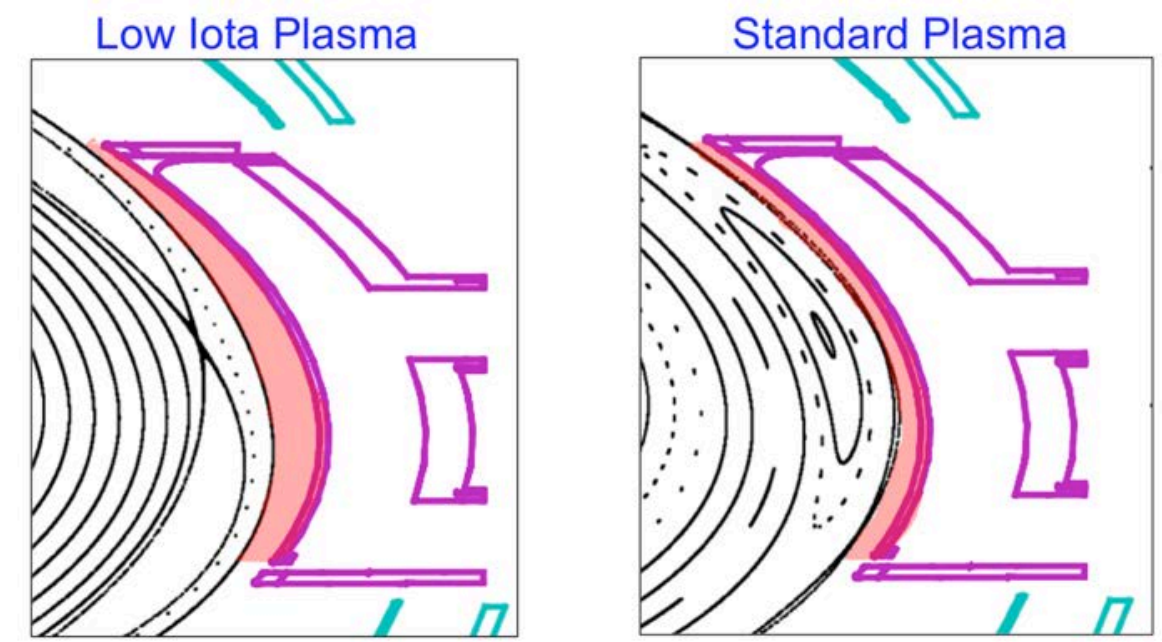

FIGURE 10 : Illustration of the variation in plasma-strap distance between the low iota and standard configuration.

addition, we need to take into account the power flux from the plasma to the antenna box. In parking position an average power of $14 \mathrm{~kW}\left(50 \mathrm{~kW} / \mathrm{m}^{2}\right)$ must be removed for the full plasma duration. This value can increase significantly at the carbon limiters of the antenna box if the antenna is moved towards the LCMS especially if magnetic islands would be present close to the antenna box. Modeling of the power flow towards the plasma facing surfaces of the antenna box will be performed and will determine the detailed 3D shape of the graphite limiter at the edges of the antenna box. ANSYS calculations will deliver surface temperatures of the critical components for a given water flow, and will determine the acceptable positions of the antenna for all plasma configurations. Thermocouples in the limiter surfaces open the possibility of implementing a feedback system. A significant part of the RF power will also be dissipated in the capacitors, and therefore they will be connected to separate water cooling circuits.

A calculation of the heat load on the antenna from convective losses is shown in Fig. 10 for three different radial positions of the antenna with the plasma corresponding to the standard magnetic field configuration. As the antenna is moved in, we clearly see the appearance of extra loading on the lower and upper boundaries of the antenna. This is due to the presence of islands close to the antenna, causing an ergodization of the magnetic field in the edge. To avoid this, one should therefore also take into account the extra heat load caused by islands.

\section{Grounding of the Antenna Box}

The antenna box should be grounded to the wall of the port duct to avoid RF resonances excitation in the space between the port duct and the antenna plug-in behind the antenna box [13]. The antenna structure must be encapsulated in a metal enclosure until the location of the short circuit.

\section{Safety System}

Operation of the antenna system requires the monitoring of all temperatures at the antenna head and flow control in the cooling water circuits. Input from the RF power measurement is necessary for the operation of the generators. 


\section{ANTENNA DIAGNOSTIC SYSTEMS}

The diagnostics that will be integrated into the antenna head are :

- Power detector at the capacitors $\left(\mathrm{U}_{\mathrm{RF}}\right.$ and $\left.\mathrm{I}_{\mathrm{RF}}\right)$

- Thermocouples at the antenna guard limiter (type $\mathrm{K}$ for temperatures up to $1250^{\circ} \mathrm{C}$

- Pt100 sensors at the antenna box and coaxial tubes (temperatures up to $200^{\circ} \mathrm{C}$ )

- Electrical probes in the guard limiters (plasma parameter)

- Pressure gauges for vacuum control (pressure control in the rear side antenna port)

- Probes for the RF voltage und current in the antenna box.

In addition, a dedicated gas injection system close to the antenna box for optimizing RF coupling under all circumstances will be implemented.

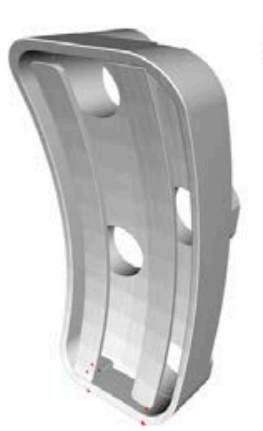

$+275 \mathrm{~mm}$

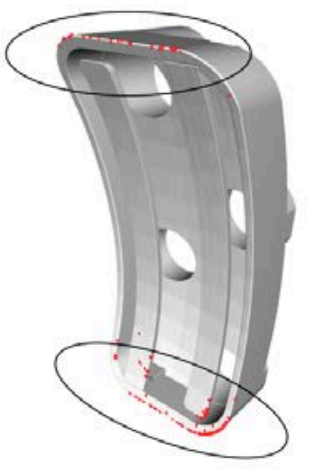

$+280 \mathrm{~mm}$

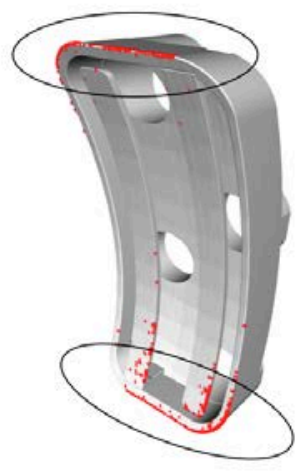

$+285 \mathrm{~mm}$

FIGURE 11: Plasma load on the edges on the antenna from escaping fast particles. The antenna shape is aligned to the shape of the LCMS of the plasma. When moving the antenna closer to the plasma, an additional load appears at the top and bottom of the antenna box, due to the presence of ergodized magnetic lines at the location of island structures. This effect will have to be taken account in shaping the antenna.

\section{REFERENCES}

[1] J.Nührenberg et al., "Overview of Wendelstein 7-X Theory", Trans. Fusion Technology 27, 71 (1995)

[2] R.C. Wolf, et al., "A stellarator reactor based on the optimization criteria of Wendelstein 7-X," Fusion Eng. Design $83,990(2008)$

[3] A.Messiaen et al., "Coupling and matching study of the ICRF Antenna for W7-X", this conference.

[4] A.Messiaen et al., Nucl. Fusion 50 (2010) 025026

[5] D.Van Eester et al., Plasma Phys. Control. Fusion 40 (1998) 1949

[6] P.Lamalle, LPP-ERM/KMS Lab Report 101 (1994)

[7] F.Durodié et al., "RF Optimization of the Port Plug Layout and Performance Assessment of the ITER ICRF Antenna", Proc. $24^{\text {th }}$ IAEA Fusion Conf, San Diego, Paper ITR/P1-08

[8] V.Lancelotti et al., Nucl. Fusion 46 (2006) S476

[9] CST Microwave Studio, User Manual Version 2009, Sep. 2008, CST AG, Darmstadt, Germany, www.cst.com

[10] A.Messiaen et al., Fusion Eng and Design, 86 (2011) 855-859

[11] F.Durodié, M.Vervier, Fusion Technology 1992 (Proc. $17^{\text {th }}$ SOFT, Roma) p 477 (1993)

[12] A.Messiaen et al., Nucl. Fusion 49 (2009) 055004

[13] P. Dumortier et al., ITER ICRH antenna grounding options”, Fusion Eng. Des. (2013), in print 